\title{
Objects of attention, objects of perception
}

\author{
JUDITH AVRAHAMI \\ Hebrew University, Jerusalem, Israel
}

\begin{abstract}
Four experiments were conducted, to explore the notion of objects in perception. Taking as a starting point the effects of display content on rapid attention transfer and manipulating curvature, closure, and processing time, a link between objects of attention and objects of perception is proposed. In Experiment 1 , a number of parallel, equally spaced, straight lines facilitated attention transfer along the lines, relative to transfer across the lines. In Experiment2, with curved, closed-contour shapes, no "sameobject" facilitation was observed. However, when a longer time interval was provided, in Experiment 3, a same-object advantage started to emerge. In Experiment 4, using the same curved shapes but in a nonspeeded distance estimation task, a strong effect of objects was observed. It is argued that attention transfer is facilitated by line tracing but that line tracing is encouraged by objects.
\end{abstract}

The effects of location on selective attention have long been demonstrated (e.g., B. A. Eriksen \& C. W. Eriksen, 1974; Posner, 1980; Tsal \& Lavie, 1988). Attention transfer has been shown to require a longer time the greater the distance traversed (Posner, 1980), and attention allocation to a target defined by any of the features of location (e.g., C. W. Eriksen \& Hoffman, 1972), color, or shape (e.g., Tsal \& Lavie, 1988) has been shown to emanate to its vicinity. Thus, distractors located close to the target cause more interference than distractors located farther away (C. W. Eriksen \& Hoffman, 1972), and relevant stimuli near the target are more often identified than other stimuli in a display (Tsal \& Lavie, 1988).

Recently, however, several studies have shown that other factors, having to do with the layout of a display, may also have an effect on the way attention is allocated, at times conflicting with predictions based on pure location considerations (e.g., Baylis \& Driver, 1992; Duncan, 1984; Egly, Driver, \& Rafal, 1994; Kahneman \& Henik, 1981; Kramer \& Jacobson, 1991; Treisman, Kahneman, \& Burkell, 1983; Vecera \& Farah, 1994). Treisman et al. (1983) showed that the latency of reading a single word and detecting a gap in a frame is longer when the frame and word are separate than when the frame surrounds the word. This was the case even though the distance between word and gap was kept constant. Kramer and Jacobson (1991) and Baylis and Driver (1992) used a focused attention task in which a target is categorized while distractors are ignored. Both studies showed that factors that are known to

This paper was written while the author was a visiting scholar at the Department of Psychology, University of Chicago. The author thanks Robert Gordon and Morris Goldsmith for their helpful comments on an earlier draft. Correspondence should be addressed to J. Avrahami, The Goldie Rotman Center for Cognitive Science and Education, Hebrew University, Jerusalem, Israel (e-mail: judav@vms.huji.ac.il).

-Accepted by previous editor, Myron L. Braunstein determine perceptual grouping in a display (e.g., color, good continuation) affect the degree of interference in the same manner as does distance and can, at times, even override distance effects. Duncan (1984) and, later, Vecera and Farah (1994) showed that two judgments concerning either a box or a line were more efficiently performed than two judgments of which one concerned the box and another concerned the line, even when the line was superimposed on the box. They concluded that attention is, at least in some cases, object based. Thus, the notion of perceptual objects is used to explain mechanisms of attention.

Of particular importance to the notion of object-based attention is a study by Egly et al. (1994). Egly et al. used a cued detection paradigm to test the effect of cue and display on attention. Their basic display consisted of two, parallel, outline rectangles; the cue was a change in brightness of a U-shaped portion of the end of one of the rectangles, and the target was a solid square that appeared at an end of a rectangle. On valid trials, the target appeared at the cued end of the cued rectangle. On invalid trials, the target appeared either at the uncued end of the cued rectangle or at an equidistant end of the uncued rectangle. Egly et al. found that costs for invalidly cued targets were higher for targets on the uncued rectangle than for targets on the cued rectangle. They concluded that an objectbased component of visual attention was responsible for this result. It is on the findings reported by Egly et al. that I wish to focus in the present paper.

While there is no denying that each rectangle in the display used by Egly et al. (1994) can be regarded as a perceptual object of "good form" and perfect continuation, there may be other, more basic, properties of the display that could account for the results. More specifically, apart from being good objects, the elongated rectangles (with a ratio of about 1 to 7 ), clearly define a general directiona grain, as it were-in the display. It may well be the case that the long straight lines, all in one direction, are respon- 
sible for the relative ease of attention transfer along the direction of the rectangles relative to attention transfer against them. In other words, maybe not all other features that are necessary to define the stimuli as perceptual objects - that is, closure and proximity - are necessary for the effects observed. To test this possibility, the Egly et al. study was replicated, using a slightly different display.

In the first experiment to be reported here, the basic display consisted of unconnected parallel lines equally spaced from one another. As in Egly et al.'s (1994) experiments, the cue was a U-shaped contour of different brightness from the lines and the target was a solid square in the same brightness as the lines. The lines were of the same length as the lines of the rectangles in the Egly et al. study.

\section{EXPERIMENT 1}

Experiment 1 used a cued detection paradigm in which the cue was valid in most of the trials and invalid in others, with a number of catch trials. Of the invalid trials, the target lay farther away along the direction of the lines on half of the trials and at the same distance from the cue but across the lines from it on the other half. The question addressed was whether attention transfer to invalidly cued targets removed from the cue along the lines of the display would be faster than it would be to targets lying across the lines from the cue.

\section{Method}

Participants. Twenty-three undergraduate students participated in the experiment in partial fulfillment of a course requirement. All had normal or corrected-to-normal vision.

Stimuli. The stimuli included seven parallel lines, a cue, and a target. With participants viewing the screen from a distance of approximately $60 \mathrm{~cm}$, the lines subtended $11.4^{\circ}$ in length and were spaced $1.9^{\circ}$ apart; they were drawn in gray with a stroke of 4 pixels. The cue was a black $U$ shape (with a stroke of 4 pixels), and the target was a solid gray box. The size of both cue and target was $1.1^{\circ} \times 1.1^{\circ}$. The center of the cue and the center of an invalidly cued target were $5.7^{\circ}$ apart. All stimuli were presented via a Macintosh IICX computer, with a screen size of $17 \times 23 \mathrm{~cm}$. Since the lines were $12 \mathrm{~cm}$ long, $2.5 \mathrm{~cm}$ were left above and below the lines in the vertical condition and $5.5 \mathrm{~cm}$ were left on both sides in the horizontal condition.

Design. Out of all trials, $67 \%$ had valid targets (targets in the same location as the cue), $11 \%$ were invalid with target along the directıon of the lines from the cue, $11 \%$ were invalid with target across the lines from the cue, and $11 \%$ were catch trials (no target). For each of these trials, the lines were either vertical or horizontal. The cue could appear in one of four loçations, equidistant from the center of the display, between two lines. The opening in the U-shaped cue changed sides in accordance with the direction of the lines and the location of the cue. For example, when the lines were vertical and the cue was at the bottom (either right or left), the cue opened upward, as a normal U. But when the cue was at the top (again, either right or left) the $U$ was inverted. Cues open to the right or to the left were similarly used in the horizontal display. The adjustment of cue shape was done to parallel, as closely as possible, the direction of the cue in Egly et al.'s (1994) study.

Procedure. Every participant first performed a short practice block of 24 trials: 18 of the trials were valid, 2 invalid across the lines, and 2 catch trials. Both types of display (vertical and horizontal) and all cue locations were equally represented in this block. After practice, every participant performed one experimental block of 216 trials, of which 144 were valid trials, 24 along-the-lines invalid trials, 24 across-the-lines invalid trials, and 24 catch trials. The order of trials within the block was randomized.

The sequence in a single trial was as follows (see Figure 1): First, only the lines appeared for $1,050 \mathrm{msec}$, then the cue flashed for $105 \mathrm{msec}$, then again the lines, and, after $210 \mathrm{msec}$, the target appeared (unless it was a catch trial, in which case no target appeared). The participants were instructed to press the space bar as soon as they spotted the target. Once responding, a new trial began, following an intertrial blank of $510 \mathrm{msec}$. A point was made that the participants should not press the bar when no target appeared. In that case, 2,220 msec elapsed before a new trial began. The experimental block was divided into six subblocks, allowing the participants a short rest after every 36 trials.

\section{Results and Discussion}

Median response time (RT) was calculated for every participant for each of the experimental conditions. The mean of the median RT was $355 \mathrm{msec}$ for valid targets and $369 \mathrm{msec}$ for invalid targets, yielding a validly cued target advantage of $14 \mathrm{msec}$. The difference was significant $[t(22)=3.48, S D=20.12, p=.002]$. The proportion of catch-trial errors was .04 .

The difference of interest - the advantage of invalidly cued targets lying along the lines of the display compared with invalidly cued targets lying across the lines-was $11 \mathrm{msec}[t(22)=2.90, S D=18.72, p=.008]$. This difference is similar to that observed by Egly et al. $(13 \mathrm{msec})$ for the equivalent comparison, even though the distance between cue and invalid targets was smaller here.

The results show that attention transfer may be encouraged by lines in the display and is hindered if having to go "against the grain." At the same time, the results clearly demonstrate that neither closure nor proximity is a prerequisite for such a facilitation to occur.

While the results show that proper objects of perception are not necessary for the facilitation observed by Egly et al. (1994), they did not rule out the possibility that such objects are sufficient for a similar facilitation. Indeed, effects of closure on attention have been shown before. For example, in one manipulation, Kramer and Jacobson (1991) showed that distractor lines, flanking a central target line, have a stronger effect on target judgment when connected to the target than when they are either connected to lines on the periphery or not connected at all.

To test the effect of closure, a new replication of Egly et al.'s (1994) task was conducted. This time, closedcontour objects were used, but the objects were curved in such a way that neither the same-object invalid targets nor the different-object invalid targets were favored by an overall direction of the display. To achieve this, Egly et al.'s rectangles were "bent," as it were, to a shape that will henceforth be called ribbons (see Figure 2). Again, the cue was U-shaped, but now consisting of a change in the end portion of the contour of one ribbon. A validly cued target appeared at that end of the cued ribbon, and an invalidly cued target appeared either at the other end of the cued ribbon or at the end of the uncued ribbon. 
Preparation
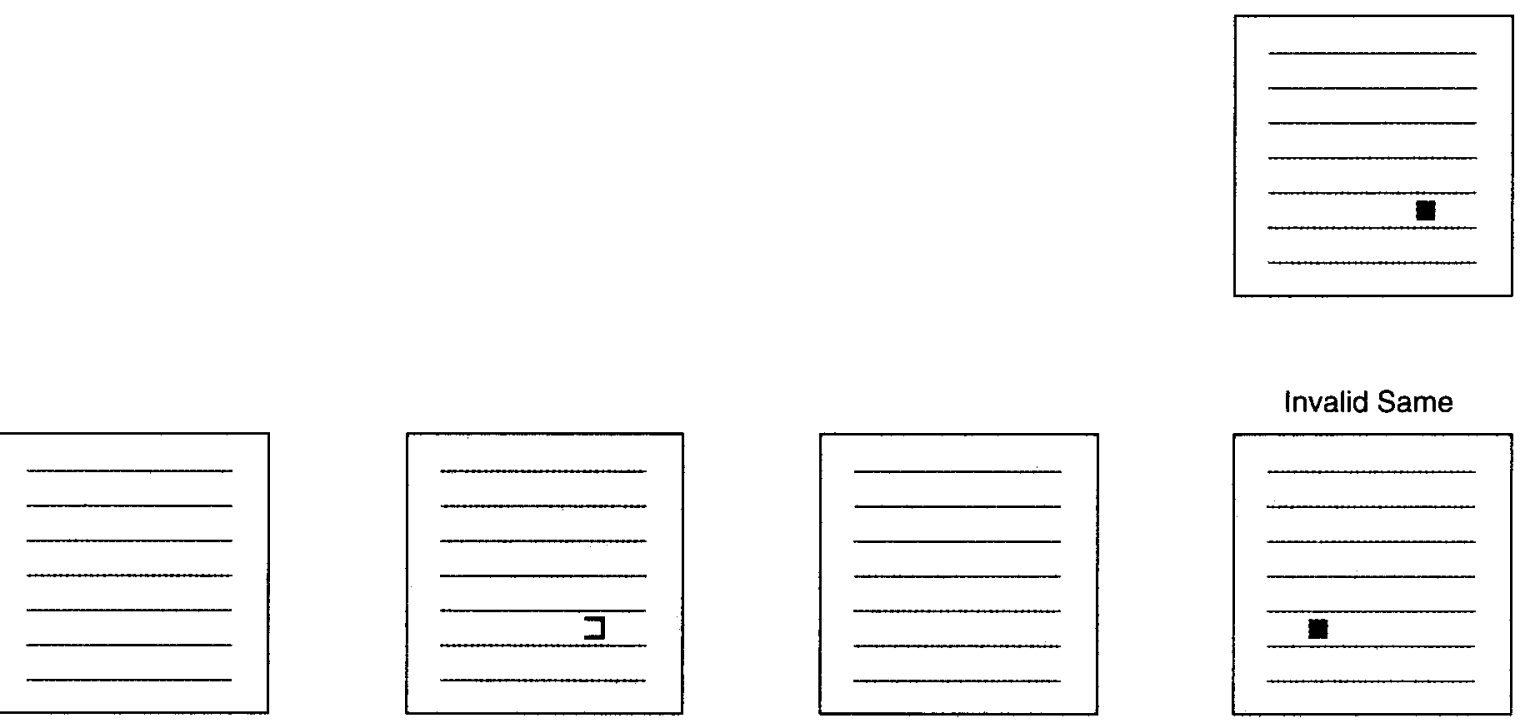

Invalid Same

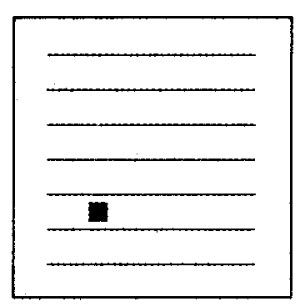

Invalid Different

$1050 \mathrm{~ms}$.

$105 \mathrm{~ms}$

$210 \mathrm{~ms}$.

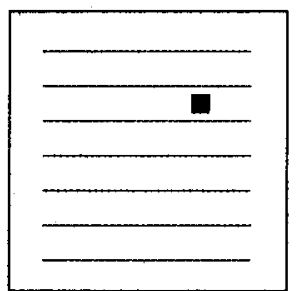

$<2220 \mathrm{~ms}$.

Figure 1. The sequence of events in Experiment 1.

The distance between the ribbons was manipulated in a manner of a study by Vecera (1994). Vecera replicated the experiments of Egly et al. (1994) but manipulated the distance between rectangles and found that distance affected the size of the same-object effect of the rectangles. In the present experiment, in one third of the trials, both types of invalidly cued targets were equidistant from the cue, whereas, in the other two thirds, the uncued-ribbon target was either one step or two steps closer to the cue than the cued-ribbon target (for more detail, see the Method section). This manipulation was introduced to prepare for the possibility that an object-based facilitation was found. In that case, a possible reduction of the effect, with change of distance, would be of interest.

In Experiments 2A, 2B, and 2C, a cued detection paradigm was used again, but this time the display consisted of two ribbons. Following a cue at the end of one ribbon, a target appeared at one of three locations: at the same end, at another end of the same ribbon, or at the end of another ribbon. Some trials were catch trials, in which no target appeared. The design and procedure of these experiments were very similar to those of Experiment 1 . To anticipate, hardly any same-object facilitation was found, but, since a lack of significant effect might have resulted from the particular contrasts in the display or from lack of statistical power, three versions of this experiment were conducted (Experiments 2A, 2B, and 2C), with only minor differences between them.

\section{EXPERIMENT 2A}

\section{Method}

Participants. Nineteen undergraduate students participated in the experiment in partial fulfillment of a course requirement. All had normal or corrected-to-normal vision.

Stimuli. The stimuli were two ribbons, a cue that consisted of blackening three sides of an imaginary outline square that overlapped one end of a ribbon, and a target that was a solid box located at the end of a ribbon. The stroke of the ribbons and the cue was 4 pixels, as in Experiment 1. Each ribbon could fit in an imaginary rectangle of $6.7^{\circ} \times 4.0^{\circ}$ of visual angle. The ribbons themselves sub- 


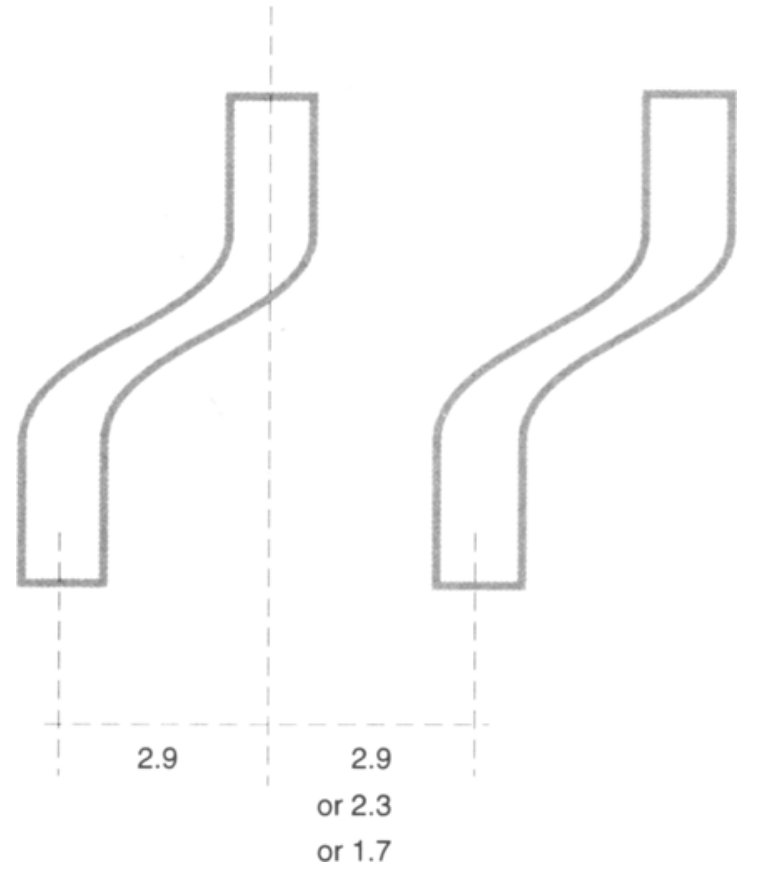

Figure 2. Ribbons; the basic display of Experiment $2 \mathrm{~A}$ (measure indications were, of course, absent).

tended $1.1^{\circ}$ in width. The cue and target were the same as those of Experiment 1 -that is, the cue was a black $U$ shape, and the target was a gray solid box, both subtending $1.1^{\circ} \times 1.1^{\circ}$ of visual angle. The stimuli were thus quite similar to those used by Egly et al. (1994). The invalidly cued target on the uncued ribbon was either equidistant to the cue as the invalidly cued target on the same ribbon or one or two steps closer to it. The distances between the center of the cue and the center of an invalidly cued target lying at the other end of the same ribbon, given in coordinates, were $5.5^{\circ}$ along one coordinate and $2.9^{\circ}$ along the other. The distance between the boxes on the same ribbon thus subtended $6.2^{\circ}$. Distance was manipulated on the coordinate perpendicular to the direction of the ribbons: In the onestep-closer condition, the distance between cue and target on the uncued ribbon (on that coordinate) was $2.3^{\circ}$; in the two-steps-closer condition, it was $1.7^{\circ}$, instead of the $2.9^{\circ}$ in the equidistant condition (again see Figure 2).

In order to ensure that the shift of attention along a cued ribbon would not always result in a shift to the same direction, the ribbons were bent to one side half of the time and to another the other half.

Design. The design and procedure were similar to those of Experiment 1 . Out of all trials, $67 \%$ had valid targets (targets in the same location as the cue), $11 \%$ were invalid with target at the other end of the same ribbon as the cue, $11 \%$ were invalid with target at the end of the other ribbon, and $11 \%$ were catch trials (no target). For each of these trials, the ribbons were either vertical or horizontal. For the vertical display, the cue was either at the top or at the bottom; for the horizontal display, the cue was either right or left. Both ribbons in a display were bent to either one side or the other and were one of three distances apart.

Procedure. Every participant first performed a short practice block of 28 trials consisting of 16 valid trials, 4 same-ribbon invalid trials, 4 different-ribbon invalid trials, and 4 catch trials, with all three distances, both bends, and all possible cue locations represented in this block. Following the practice block, the participants performed the experimental block of 216 trials, of which 144 were valid trials, 24 same-object invalid trials, 24 different-object invalid trials, and 24 catch trials. The order of trials within the block was randomized. The block was divided into three subblocks, allowing the participants a short rest after every 72 trials.

The sequence in a single trial was very similar to that of Experiment 1 (see Figure 3): First, the empty ribbons appeared for $1,050 \mathrm{msec}$, then the cue (i.e., the blackening of an end of one ribbon) flashed for $105 \mathrm{msec}$, then again the empty ribbons, and, after $210 \mathrm{msec}$, the target appeared (unless it was a catch trial, in which no target appeared). The participants were instructed to press the space bar as soon as they spotted the target. Once a response had been made, a new trial began following an intertrial blank of $510 \mathrm{msec}$. A point was made that the participants should not press the bar when no target appeared. In that case, $2,220 \mathrm{msec}$ elapsed before a new trial began.

\section{Results}

Median RT was calculated for every participant in each of the experimental conditions. In the equidistant condition, the mean of the median RT was $323 \mathrm{msec}$ for valid targets and 362 msec for invalid targets; the valid target advantage was thus $39 \mathrm{msec}$. This advantage was highly reliable $[t(18)=4.31, S D=38.71, p<.001]$. The proportion of catch-trial errors was .12. As to the measure of interest, concerning same versus different objects, the advantage of same-ribbon invalid target over differentribbon invalid target was $-1 \mathrm{msec}$. This effect was not only unreliable but also in the opposite direction. ${ }^{1}$

Whereas the effect of invalid cue observed in the present experiment $(39 \mathrm{msec}$ ) is similar to that observed by Egly et al. $(1994 ; 40 \mathrm{msec})$, the effect of greater interest-the same-object advantage in the equidistant conditionwas nonexistent. To make sure that the disappearance of the same-object effect did not result from the particular contrast of the contour of the ribbons (i.e., from the lines of the ribbons failing to attract attention because of being both pale and curved) or from lack of statistical power, two variations on this experiment were conducted.

\section{EXPERIMENT 2B}

\section{Method}

Eighteen undergraduates, drawn from the same population as Experiments $\mathrm{I}$ and $2 \mathrm{~A}$, participated in this experiment. The design was the same as in Experiment $2 \mathrm{~A}$. The stimuli were similar to those of Experiment $2 \mathrm{~A}$, only the lines of the ribbons were thinner (with stroke of 2 pixels) and black, and the cue consisted of thickening of the end of one ribbon to a stroke of 6 pixels. As to procedure, again, every participant first performed a short practice block of 28 trials and then an experimental block of 216 trials.

\section{Results}

Median RT was calculated for every participant in each of the experimental conditions. In the equidistant condition, the mean of the median RT was $338 \mathrm{msec}$ for valid targets and $382 \mathrm{msec}$ for invalid targets; the valid target advantage was thus $44 \mathrm{msec}$. This advantage was highly reliable $[t(17)=3.22, S D=59.03, p=.005]$. The proportion of catch-trial errors was .10. As to the measure of interest, concerning same versus different objects, the 
Preparation

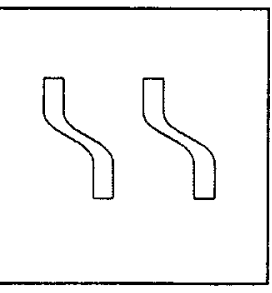

$1050 \mathrm{~ms}$.

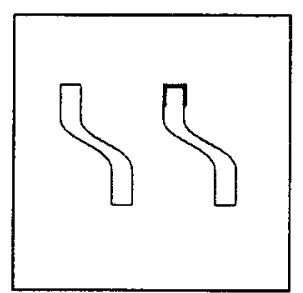

$105 \mathrm{~ms}$.
Intermediate
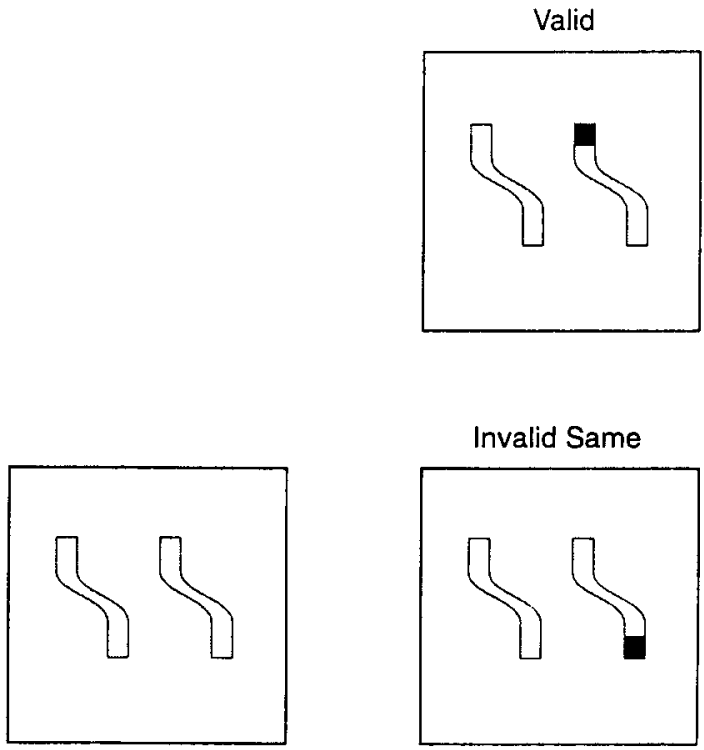

Invalid Different

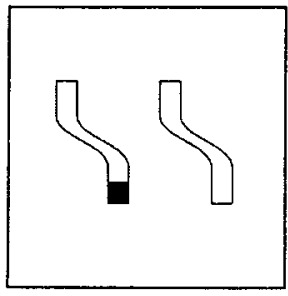

$<2220 \mathrm{~ms}$.

Figure 3. The sequence of events in Experiments 2A, 2B, and 2C.

advantage of same-ribbon invalid target over differentribbon invalid target was $2 \mathrm{msec}$. This effect was small and unreliable $[t(17)=0.19, S D=41.52, p=.854] .^{2}$

\section{EXPERIMENT 2C}

\section{Method}

Fourteen undergraduates, drawn from the same population as in Experiments 1,2A, and 2B, participated in this experiment. The design was identical and the procedure and stimuli almost identical to those of Experiments $2 \mathrm{~A}$ and $2 \mathrm{~B}$. In this experiment, the participants performed only the experimental block and in one go. The lines of the ribbons in this experiment were thin (stroke of 1 pixel) and a darker gray, as was the target. The cue was again a thickening of the end of a ribbon to a stroke of 2 pixels.

\section{Results}

Median RT was calculated for every participant in each of the experimental conditions. In the equidistant condition, the mean of the median RT was $338 \mathrm{msec}$ for valid targets and $385 \mathrm{msec}$ for invalid targets; the valid target advantage was thus $47 \mathrm{msec}$. This advantage was highly reliable $[t(13)=4.70, S D=37.32, p<.001]$. The proportion of catch-trial errors was .07. As to the measure of interest, concerning same versus different objects, the advantage of same-ribbon invalid target over differentribbon invalid target was $6 \mathrm{msec}$. This effect was still small and unreliable $[t(13)=0.67, S D=33.47, p=.514] .^{3}$

\section{Discussion}

The results are very surprising: Why should the "bending" of the rectangles disrupt the facilitation of attention transfer observed by Egly et al. (1994)? Is it because the display lacked a clear definition of direction? Or is it that curved objects require a longer time to emerge? It has been shown by Jolicoeur, Ullman, and Mackay (1986, 1991) that voluntary line tracing is slower the more curved the line. Could it be that, for an object to affect performance, its lines have to be traced first and that, since tracing of curved lines requires more time than that of straight lines, the tracing was not completed? If this is the case, 
then a longer interval between cue and target may reveal a same-object advantage for the ribbons.

Another "ribbons" experiment was conducted, in which the interval between cue and target was manipulated. In one condition, the interval was the same as in the previous experiments $(210 \mathrm{msec})$; in another condition, it was twice as long $(420 \mathrm{msec})$. This variable was manipulated within participants. In view of the previous finding that the different distances between the ribbons did not systematically affect performance, and to ensure that the distance manipulation did not, in itself, interfere with the objects' effects, only the equidistant condition was used in Experiment 3.

\section{EXPERIMENT 3}

In Experiment 3, every participant performed one block with a short stimulus onset asynchrony (SOA) and one with a long SOA. The order of the blocks was counterbalanced between participants.

\section{Method}

Participants. Twenty undergraduate students participated in the experiment either as paid volunteers or in partial fulfillment of a course requirement. All had normal or corrected-to-normal vision.

Stimuli. The stımuli were identical to those of Experiment 2C.

Design. The design and procedure were similar to those of Experiment 1 . Out of all trials, $67 \%$ had valid targets (targets in the same location as the cue), $11 \%$ were invalid with target at the other end of the same ribbon as the cue, $11 \%$ were invalid with target at the end of the other ribbon, and $11 \%$ were catch trials (no target). For each of these trials, the ribbons were either vertical or horizontal. For the vertical display, the cue was either at the top or at the bottom; for the horizontal display, the cue was elther right or left. Both ribbons in a display were bent to either one side or the other.

Procedure. Every participant first performed a short practice block of 36 trials consisting of 24 valid trials, 4 same-ribbon invalid trials, 4 different-ribbon invalid trials, and 4 catch trials, with both bends, both horizontal and vertical, and all possible cue locations represented in this block. Following the practice block, the participants performed two experimental block of 144 trials each, of which 96 were valid trials, 16 same-object invalid trials, 16 differentobject invalid trials, and 16 catch trials. The order of trials withın the block was randomized. The blocks were divided into two subblocks, allowing the participants a short rest after every 72 trials.

The sequence in a single trial was very similar to that in the previous experiments with one exception: After disappearance of the cue, the empty ribbons stayed on for $315 \mathrm{msec}$ in the practice block trials, for $210 \mathrm{msec}$ in the short-SOA block trials, and for $420 \mathrm{msec}$ in the long-SOA block trials.

\section{Results}

Median RT was calculated for every participant in each of the experimental conditions. In the short-SOA condition, the mean of the median RT was $322 \mathrm{msec}$ for valid targets and $359 \mathrm{msec}$ for invalid targets; in the long-SOA condition, the mean of the median RT was $352 \mathrm{msec}$ for valid targets and $376 \mathrm{msec}$ for invalid targets. The valid target advantage was thus $37 \mathrm{msec}$ in the short-SOA condition and $24 \mathrm{msec}$ in the long-SOA condition. An analysis of variance (ANOVA) with validity and SOA as within- participants variables revealed significant main effects of both validity $\left[F(1,19)=28.88, M S_{\mathrm{e}}=641.53, p<\right.$ $.001]$ and SOA $\left[F(1,19)=9.76, M S_{\mathrm{e}}=1,123.30, p=\right.$ $.006]$. Although the interaction of validity and SOA was not significant $\left[F(1,19)=2.19, M S_{\mathrm{e}}=337.87, p=.155\right]$, it is interesting to note that the validity effect was greater in the short-SOA condition than in the long-SOA condition. The proportion of catch-trial errors was .04 in the short-SOA condition and .01 in the long-SOA condition.

As to the measure of interest, concerning same versus different objects, the advantage of same-ribbon invalid target over different-ribbon invalid target was $-6 \mathrm{msec}$ in the short-SOA condition and $12 \mathrm{msec}$, in the long-SOA condition. An ANOVA with same-object versus differentobject invalid targets and SOA as within-participants variables again revealed an almost significant effect of SOA $\left[F(1,19)=4.01, M S_{\mathrm{e}}=1,498.13, p=.060\right]$ and no main effect of same-object versus different-object $(F<1)$, but an interaction that approached significance $[F(1,19)=$ $\left.2.80, M S_{\mathrm{e}}=524.91, p=.111\right]$. A $t$ test of the same-object advantage conducted separately for each SOA condition showed the (negative) effect in the short-SOA condition to be nonsignificant $[t(19)=0.88, S D=30.16, p=.393]$ but that in the long-SOA condition to be quite close to significance $[t(19)=1.99, S D=25.33, p=.062]$.

\section{Discussion}

Though not reaching conventional levels of significance, the results indicate that when the ribbons have a longer time to be traced, attention transfer to an invalidly cued target within a ribbon is more efficient than that to a target in a different ribbon: In the short-SOA condition, the noneffect of Experiments $2 \mathrm{~A}, 2 \mathrm{~B}$, and $2 \mathrm{C}$ was replicated, whereas an almost reliable effect was observed in the long-SOA condition. ${ }^{4}$ Since attention transfer is more efficient within a ribbon than across ribbons, when enough time is available, would this facilitation also affect later processes of perception such as, for example, distance estimation? This was tested in the next experiment. In Experiment 4 , participants were asked to judge whether a reference box, lying at the end of one ribbon (in the location of a cue in the previous experiments), was closer to a comparison box at the other end of the same ribbon (at the location of the former invalid same-ribbon target) or to a comparison box at the end of another ribbon (in the location of the former invalid different-ribbon target). Experiment 4 tested the prediction that the comparison box on the same ribbon would seem closer than that on the other.

\section{EXPERIMENT 4}

In Experiment 4, the participants' task was to judge which of a pair of comparison boxes on one side of the display was closer to a single, critical box on the other side (see Figure 4). Of the two comparison boxes, one was at the other end of the same ribbon as the critical box, and the other was at an end of a second ribbon. Thus, if 

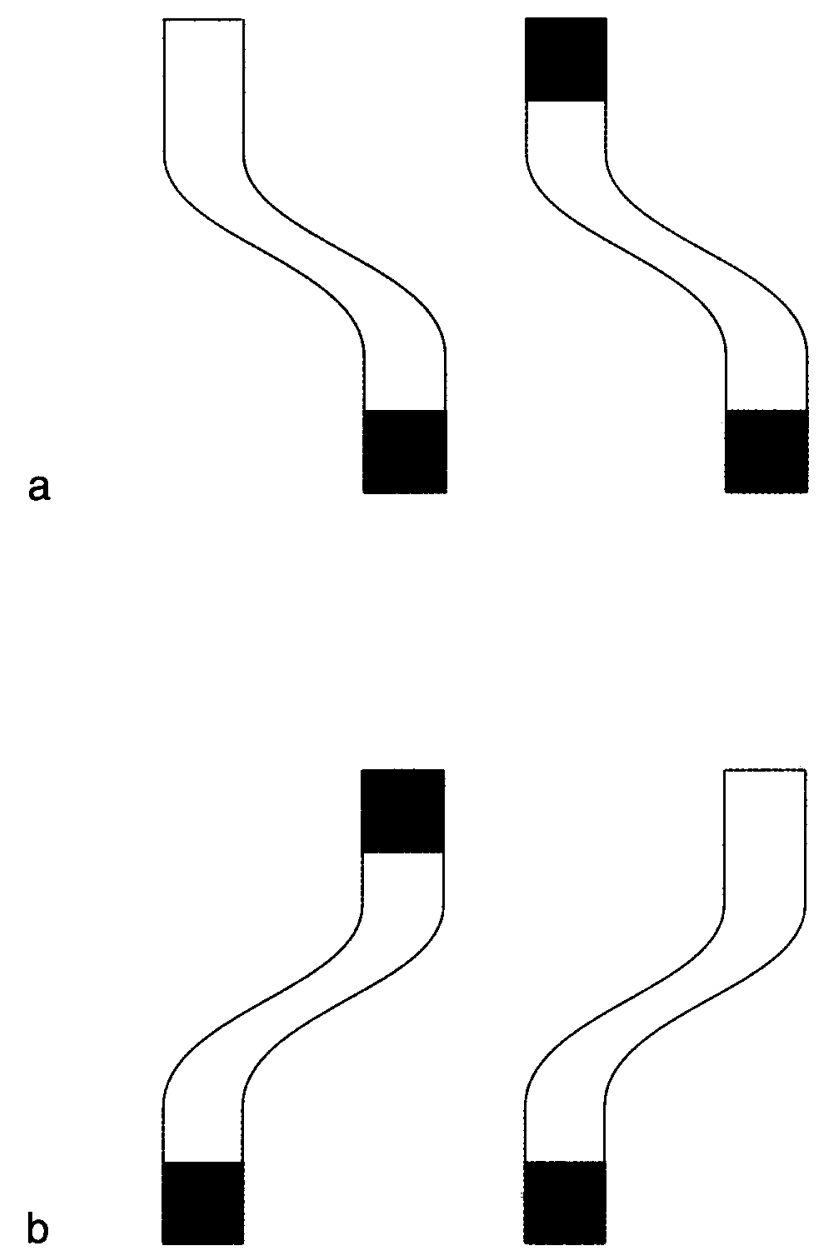

Figure 4. Two displays of ribbons with reference and comparison boxes of Experiment 4, showing the two possible bends of the ribbons. The participants judged which of the two boxes at the bottom was closer to the single box at the top.

the two boxes sharing a ribbon were grouped by virtue of their belonging to the same object, the perceived distance between them should seem smaller than the perceived distance between the critical box and the second comparison box, even if both distances were objectively the same.

\section{Method}

Participants. Sixteen undergraduate students participated in the experiment in partial fulfillment of a course requirement. All had normal or corrected-to-normal vision.

Stimuli. The ribbons in this experiment were identical to those of Experiment $2 \mathrm{C}$ - that is, each ribbon could fit in an imaginary rectangle of $6.7^{\circ} \times 4.0^{\circ}$ of visual angle, and the ribbons subtended $1.1^{\circ}$ in width drawn with a gray stroke of one pixel. The three boxes were the same as the target of Experiment $2 \mathrm{C}$ (i.e., $1.1^{\circ} \times 1.1^{\circ}$ and of the same gray). The distance between the two ribbons was again manipulated, but this time it could assume one of five equally spaced values, with boxes on the same ribbon either closer or further apart than the boxes on different ribbons. This was done to prevent participants from learning that the comparison box on the different ribbon was never further away than the box on the same ribbon with the reference box. The distance between the reference box and the comparison box on the different ribbon was always $5.5^{\circ}$ along one coordinate (along the length of the ribbons) and one of $1.7^{\circ}, 2.3^{\circ}, 2.9^{\circ}, 3.5^{\circ}$, and $4.1^{\circ}$ in the two-steps-closer, one-stepcloser, equidistant, one-step-farther-away, and two-steps-fartheraway conditions, respectively, along the second coordinate (see Figures 2 and 4 ).

The ribbons were again lying either vertically or horizontally on the screen. In the vertical condition, the critical box could be at the top, with the comparison boxes at the bottom, or vice versa; in the horizontal condition, the critical box could be on the left and the comparison boxes on the right, or vice versa.

The ribbons were again bent in one direction (as in Figure $4 a$ ) or in another (as in Figure 4b). This ensured that judging the comparison box on the right (or the left) or at the top (or the bottom) to be closer did not always coincide with judging the same-ribbon box to be closer (or farther away). To take, for example, the layouts depicted in Figure 4, responding that the right box was closer to the top box would mean that the same-ribbon box was judged to be closer for the display in Figure 4a but that the different-ribbon box was judged to be closer for the display in Figure $4 \mathrm{~b}$. This manipulation fully controlled for any direction or keypress bias.

Procedure. In each trial, the display stayed on the screen until either the participant responded or $7,500 \mathrm{msec}$ had elapsed. Timeouts were introduced to prevent the participants from attempting to actually measure the distances. No feedback was provided. Trials were blocked by the location of the critical box: top, bottom, left, or right. ${ }^{5}$ Each block consisted of 12 trials, of which 4 were in the equidistant condition and 2 were in each of the other four distance conditions. Withın each distance, the ribbons bent one way on half the trials and the other way on the other half. The order of trials within a block was randomized.

To respond, the participants used the numeric keypad, pressing, in the vertical (top and bottom) blocks, "4" for "left box closer," "6" for "right box closer," and "5" for "equidistant"; in the horizontal (left and right) blocks, they pressed " 8 " for "top box closer," "2" for "bottom box closer," and "5" for "equidistant." The reason for having twice as many equidistant trials was to ensure that each response was equally likely.

Every participant performed all four blocks twice, thus performing 96 trials, in 32 of which the same-ribbon comparison box and the different-ribbon comparison box were equally distant from the critical box and in 64 of which the different-ribbon comparison box was either closer or farther apart. The order of the blocks was balanced across particıpants.

\section{Results}

The measure used to assess the effect of objects on the judgment of distance was the number of errors made in the different conditions. Errors were classified into those that could be explained by an object bias and those that could not. Errors that could be explained by an object bias included (1) in the equidistant condition, erroneously judging the same-ribbon comparison box to be closer than the other to the critical box, and (2) in the differentribbon-closer conditions, erroneously judging the sameribbon comparison box to be closer to the critical box than the different-ribbon comparison box or judging them to be equidistant.

Note that, in the different-ribbon-farther-away conditions, the correct answer was "same ribbon closer"; hence, none of the errors made in these conditions could be explained by an object bias. There was thus an equal number of trials in which the participants could make explainable and nonexplainable errors. 
Table 1

Mean Distance Judgment Errors in Experiment 4

\begin{tabular}{|c|c|c|c|c|}
\hline \multirow[b]{2}{*}{ Condition } & \multicolumn{2}{|c|}{ Mean Errors } & \multirow[b]{2}{*}{ Difference } & \multirow[b]{2}{*}{$t(15)$} \\
\hline & Explainable & Nonexplainable & & \\
\hline Equidistant & 11.62 & 4.44 & 7.18 & $5.44^{* * *}$ \\
\hline One Step & & & 5.12 & $3.66^{* *}$ \\
\hline Different closer & 9.50 & n.a. & & \\
\hline Different farther & n.a. & 4.38 & & \\
\hline Two Steps & & & 3.44 & $2.82^{*}$ \\
\hline Different closer & 5.25 & n.a. & & \\
\hline Different farther & $\mathbf{n}$ & 1.81 & & \\
\hline Overall & 26.37 & 10.63 & 15.74 & $4.68^{* *}$ \\
\hline
\end{tabular}

Note-n.a., not applicable (i.e., errors of this type could not be made in this condition). $\quad{ }^{*} p<.05 . \quad{ }^{* *} p<.01 . \quad{ }^{* * *} p<.001$.

Mean numbers of errors in the different conditions are presented in Table 1. Overall, of 96 judgments per participant, the average number of errors was 37.00 , of which 26.37 could be explained by an object bias, whereas the remaining 10.63 could not. The difference was significant $[t(15)=4.68, S D=13.47, p<.001]$.

Object-biased judgments were most prevalent in the equidistant condition, in which, of 32 judgments made by each participant, there were on average 16.06 errors, of which 11.62 could and 4.44 could not be explained by the bias $[t(15)=5.44, S D=5.28, p<.001]$.

To compare explainable and nonexplainable errors when the boxes were not equidistant to the critical one, errors in the different-ribbon-one-step-closer condition (explainable) were compared with errors in the differentribbon-one-step-farther-away condition (nonexplainable), and errors in the different-ribbon-two-steps-closer condition (explainable) condition were compared with errors in the different-ribbon-two-steps-farther-away condition (nonexplainable). Though smaller, the difference between explainable and nonexplainable errors in the nonequidistant conditions was also significant. For the one-step distance, the number of explainable errors was 9.50 , and the number of nonexplainable errors was 4.38 . A test of the difference resulted in $t(15)=3.66, S D=$ $5.61, p=.002$. For the two-step distance, the number of explainable errors was 5.25 , and the number of nonexplainable errors was 1.81 . A test of the difference resulted in $t(15)=2.82, S D=4.87, p=.013$.

\section{Discussion}

The results present a phenomenon that amounts to a perceptual illusion. Note, by the way, that the differentribbon comparison box in Figure 4 is closer to the reference box than is the same-ribbon comparison box. This illusion shows that ribbon-like objects can indeed distort distance estimates, with targets on the same ribbon seeming closer than they really are. As expected, the effect of the same-object bias became less pronounced with distance. The effect of grouping on distance estimates has been shown before (e.g., Enns \& Girgus, 1985) and can be seen as a demonstration of Palmer's (1992) commonregion grouping principle. In the present context, it shows the strength of the effects of grouping into perceptual objects when the task encourages such grouping.

\section{GENERAL DISCUSSION}

The results of the experiments reported here expand existing data on attention shifts within a display and may provide insights into the manner in which objects emerge. Experiment 1, essentially replicating Egly et al.'s (1994) study, demonstrated that the properties of a display can affect attention to the extent that attention shift from a cue to an invalidly cued target in one part of the display may be faster than from that cue to another invalidly cued target, even when both are equidistant from the cue. At the same time, Experiment 1 showed that the properties of closure and proximity, which turned the long lines in Egly et al.'s display into proper objects, were not necessary: Similar results were obtained in their absence. The results of Experiments $2 \mathrm{~A}, 2 \mathrm{~B}$, and $2 \mathrm{C}$ showed that these properties were also not always sufficient. In the latter experiments, using bent objects, the same-object advantage was, if at all, small and unreliable. However, when a longer time interval was allowed between cue and target, as in one of the two conditions in Experiment 3, a sameobject advantage started to show. In Experiment 4, again using bent objects but in a different, nonspeeded task, the same-object advantage was manifested in full.

The results of Experiment 1 can be explained by assuming mandatory tracing operations, which involuntarily follow lines in the display to be at work. More specifically, it may be assumed that, following the appearance of a cue, lines next to it are, to use Baylis and Driver's (1992) expression, "covertly scanned." Such mandatory tracing would, in turn, facilitate attention transfer to targets lying along the traced lines but not to targets lying to the side of those lines. This analysis may easily explain the facilitation observed in Experiment 1 and some of the findings of Baylis and Driver: In a task requiring a rapid two-choice response to a target letter, when lines were added to a basic display of a target and four distractors surrounding it (as in their Experiments 5 and 6), distractors lying along the lines exerted more interference than did distractors lying orthogonal to them.

The findings of Experiment 1 are also in line with those of other recent studies, showing that certain basic properties of a display may be responsible for the effects attributed to a more general notion of objects. In two different studies, Kramer and Watson (1996a, 1996b) compared the speed of processing two ends of one object with that of processing the equally distant ends of two different objects. The objects depicted were two wrenches, of which one could have an open end and the other a bent end or one wrench could have both an open and a bent end. They found that, when the two objects were of uniform texture, participants responded more quickly if both lookedfor features were on the same object than if they were distributed between the two objects, but, when change of texture in the objects broke them into three regions, the 
same-object facilitation was gone. It is most relevant to the present study that, while the change in texture affected rapid attention transfer, it did not affect the perception of the wrenches as whole objects (as was determined by separately asking participants to judge the objects). It would be interesting to see whether a longer presentation time of the wrenches would also induce a same-object advantage in the nonuniform wrenches.

To come back to the ribbons, it is of critical importance to realize that the participants had ample time to parse the display and group it into objects before either cue or target appeared. Indeed, the $210 \mathrm{msec}$ added to the SOA seemed negligible relative to the $1,050 \mathrm{msec}$ in which the display was present prior to cue onset. Still, it was this addition to the SOA that brought about the same-object advantage, and it was, most probably, the time allowed to move attention from reference box to comparison box along the lines of a single ribbon (in Experiment 4) that yielded the shorter distance illusion.

Apparently, it is not the general scanning or grouping of a scene that yields the same-object advantage. Instead, the active line tracing that emanates from the cued location over to the favored invalid target location or from one box to the other same-ribbon box best explains the present results. This is not to say that other characteristics of the display are immaterial. The straightness of the lines in Experiment 1 undoubtedly facilitated tracing, and the closure of the ribbons in the rest of the experiments undoubtedly encouraged the tracing of the curved lines.

At the same time, the above analysis provides a glimpse into the transient nature of attentional objects. The present results suggest that attentional objects are born for the task at hand or in response to special events such as the flashing of a cue. Such objects have no existence of and by themselves, no matter how neatly closed or how perfectly isolated from other stimuli in the display.

\section{REFERENCES}

Baylis, G. C., \& Driver, J. (1992). Visual parsing and response competition: The effect of grouping factors. Perception \& Psychophysics, 51, 145-162.

DUNCAN, J. (1984). Selective attention and the organization of visual information. Journal of Experimental Psychology: General, 113, 501-517.

Egly, R., Driver, J., \& Rafal, R. D. (1994). Shifting visual attention between objects and locations: Evidence from normal and parietal lesion subjects. Journal of Experimental Psychology: General, 123, 161-177.

ENNS, J. T., \& Girgus, J. S. (1985). Perceptual grouping and spatial distortion: A developmental study. Developmental Psychology, 21, 241-246.

ERIKSEN, B. A., \& ERIKSEN, C. W. (1974). Effects of noise letters upon the identification of a target letter in a nonsearch task. Perception \& Psychophysics, 16, 143-149.

Eriksen, C. W., \& Hoffman, J. E. (1972). Temporal and spatial char- acteristics of selective encoding from visual displays. Perception \& Psychophysics, 12, 201-204.

Jolicoeur, P., Ullman, S., \& Mackay, M. (1986). Curve tracing: A possible basic operation in the perception of spatial relations. Memory \& Cognition, 14, 129-140.

Jolicoeur, P. Ullman, S., \& Mackay, M. (1991). Visual curve tracing properties. Journal of Experimental Psychology Human Perception \& Performance, 17, 997-1022.

Kahneman, D., \& Henik, A. (1981). Perceptual organization and attention. In M. Kubovy \& J. R. Pomerantz (Eds.), Perceptual organization (pp. 181-211). Hillsdale, NJ: Erlbaum.

Kramer, A. F., \& J ACOBSON, A. (1991). Perceptual organization and focused attention: The role of objects and proximity in visual processing. Perception \& Psychophysics, 50, 267-284.

Kramer, A. F., \& WATSON, S. E. (1996a). Object-based visual selection and the principle of uniform connectedness. In A. F. Kramer, G. D. Logan, \& M. G. H. Coles (Eds.), Converging operations in the study of visual selective attention (pp. 395-414). Washington, DC: Amer1can Psychological Association.

Kramer, A. F., \& Watson, S. E. (1996b, November). Perceptual representations and object-based attentional selection. Paper presented at the annual meeting of the Psychonomic Society, Chicago.

Palmer, S. E. (1992). Common region: A new principle of perceptual grouping. Cognitive Psychology, 24, 436-447.

POSNER, M. I. (1980). Orienting of attention. Quarterly Journal of Experimental Psychology, 32, 3-25.

Treisman, A., Kahneman, D., \& Burkell, J. (1983). Perceptual objects and the cost of filtering. Perception \& Psychophysics, 33, 527-532.

TSAL, Y., \& LaviE, N. (1988). Attending to color and shape: The special role of location in selective visual processing. Perception \& Psychophysics, 44, 15-21.

VECERA, S. P. (1994). Grouped locations and object-based attention: Comment on Egly, Driver, and Rafal (1994). Journal of Experimental Psychology: General, 123, 316-320.

Vecera, S. P., \& Farah, M. J. (1994). Does visual attention select objects or location? Journal of Experimental Psychology: General, 123, 146-160.

\section{NOTES}

1. With no same-object advantage in the equidistant condition, there may be little interest in what happened in the one-step-closer and the two-steps-closer conditions. Still, since the different distances may provide more statistical power, the same-object advantage was calculated again, this time for all three distances lumped together. The difference was small, $5 \mathrm{msec}$, and not significant $[t(18)=0.71, S D=32.71, p=.486]$.

2 . The same-object advantage for all three distances lumped together was $-1 \mathrm{msec}$ (i.e., in the wrong direction) and not significant.

3. The same-object advantage for all three distances lumped together was $10 \mathrm{msec}$, but still not significant $[t(13)=1.45, S D=25.44, p=$ $.171]$.

4. It would have been interesting to see whether this trend is strengthened with an even longer SOA; but, since the longer SOA in Experiment 3 was the longest possible before mechanisms of inhibition of return intervene, this could not be tested in the same speeded detection paradigm.

5. Trials were blocked in this way to simplify the instructions given to the partıcipants (e.g., "Which of the two boxes at the bottom is closer to the single box on the top?") and to limit the number of response keys used in each block to three.

(Manuscript received December 17, 1996; revision accepted for publication August 20, 1998.) 